\title{
Biologic Disease-Modifying Antirheumatic Drug Prescription Patterns Among Rheumatologists in Europe and Japan
}

\author{
Emma Sullivan · Jim Kershaw · Stuart Blackburn · Puneet Mahajan • \\ Susan H. Boklage
}

Received: February 11, 2020 / Published online: May 21, 2020

(C) The Author(s) 2020

\section{ABSTRACT}

Introduction: Tumor necrosis factor inhibitors (TNFi) are commonly used as first-line therapy (biologic disease-modifying antirheumatic drug [bDMARD] and targeted synthetic DMARD [tsDMARD]: defined as targeted therapy) for patients with moderate-to-severe rheumatoid arthritis (RA), usually combined with conventional synthetic DMARDs (csDMARDs) but sometimes as monotherapy. If treatment fails, patients cycle to another TNFi (cycling) or switch to a targeted therapy with a different mode of action (MOA; switching). The study aimed to examine prescribing patterns and reasons for current RA treatment practice in

Digital Features To view digital features for this article go to: https://doi.org/10.6084/m9.figshare.12214589.

Electronic Supplementary Material The online version of this article (https://doi.org/10.1007/s40744020-00211-w) contains supplementary material, which is available to authorized users.

E. Sullivan $\cdot$ J. Kershaw $\cdot$ S. Blackburn

Adelphi Real World, Bollington, UK

P. Mahajan

Sanofi, Bridgewater, NJ, USA

S. H. Boklage ( $\square)$

Regeneron Pharmaceuticals, Inc., Tarrytown, NY, USA

e-mail: susan.boklage@regeneron.com
Europe (EU5: France, Germany, Italy, Spain, UK) and Japan.

Methods: Data were collected from the Adelphi Disease Specific Programme ${ }^{\mathrm{TM}}$ (DSP; Q1-Q2 2017). Rheumatologists seeing $\geq 10$ (EU5) and $\geq 5$ (Japan) patients with RA a month completed Patient Record Forms. Patients $\geq 18$ years old, with RA diagnosis and complete RA-targeted therapy history were included. Patients were grouped based on firstline targeted therapy class, and on whether firstline targeted therapy was monotherapy (targeted therapy alone) or combination therapy (targeted therapy and csDMARD). Those patients receiving TNFi at first-line and with $\geq 1$ targeted therapy were classified as TNFi cyclers or MOA switchers. Univariate analysis compared factors across groups. Patient demographics and characteristics compared across groups; physician reasoning for targeted therapy change; and time to discontinuation of targeted therapy.

Results: In EU5 and Japan, respectively, 1741 and 147 patients were included; at first-line, $80.8 \%$ and $64.6 \%$ received TNFi and $76.0 \%$ and $77.6 \%$ received combination therapy. Overall in EU5, more combination therapy than monotherapy patients reached maximum csDMARD dose before first-line targeted therapy $(P<0.05)$; disease severity was higher in patients initiating TNFi versus non-TNFi $(P<0.05)$. In Japan, trends were similar but not significant. The most common reason 
physicians gave for changing therapy following first-line targeted therapy was 'secondary lack of efficacy' (EU5: 46.2\%; Japan: 53.8\%). In EU5 and Japan, respectively, of 365 and 22 patients who received second-line targeted therapy, $52.1 \%$ and $54.5 \%$ were MOA switchers. In EU5, TNFi cyclers had longer time from diagnosis to second-line targeted therapy initiation than MOA switchers $(P=0.04)$.

Conclusions: TNFis were the most commonly prescribed targeted therapy at first-line. Between 10 and $20 \%$ of patients prescribed a TNFi as first-line targeted therapy did so without concomitant csDMARD. Almost half of patients cycled to another TNFi at second-line.

Keywords: Disease-modifying antirheumatic drugs; Physician; Rheumatoid arthritis; Switching; Tumor necrosis factor inhibitor

\section{Key Summary Points}

Why carry out this study?

Combination therapy with a targeted disease-modifying antirheumatic drug (DMARD) and a conventional synthetic DMARD (csDMARD) is recommended in clinical guidelines for patients with rheumatoid arthritis (RA) who fail to respond to csDMARDs alone.

Tumor necrosis factor inhibitors (TNFi) are the routinely administered class of targeted DMARDs, following csDMARD failure. Patients experiencing inefficacy or intolerance with their first TNFi can then cycle to another TNFi or switch to another targeted DMARD with a different mode of action (MOA).

The aim of this study was to examine prescribing patterns and reasons for current RA treatment practices in Europe (France, Germany, Italy, Spain, and the UK) and Japan, given the limited information available in the literature beyond the data for the United States.

\section{What was learned from the study?}

The findings from this analysis suggest that physician treatment decision-making is informed by the available RA treatment guidelines, but also based on clinical judgement to prescribe optimized and individualized treatments for their patients.

\section{Although treatment guidelines} recommend the use of csDMARDs in combination with targeted therapy options, at least one-third of patients were administered TNFi at first line without concomitant csDMARDs. Further research is warranted to understand physicians' reasoning behind this clinical behavior.

Furthermore, almost half the number of physicians chose to cycle to another TNFi rather than switch patients to a targeted therapy with a different MOA, despite the recognized benefits of MOA switching. Further research in this field is also warranted to better understand the reasons behind these treatment patterns, and the consequences to patient outcomes thereof.

\section{INTRODUCTION}

Around $1 \%$ of the worldwide population is affected by rheumatoid arthritis (RA) [1], which is a chronic inflammatory disease characterized by joint pain, inflammation, stiffness and swelling, and destruction of articular structures [2].

Rheumatologists are equipped with multiple treatment options for the management of patients with RA, but the backbone of RA management is conventional synthetic diseasemodifying antirheumatic drugs (csDMARDs), especially methotrexate [3]. However, many patients have an inadequate response to or are intolerant of csDMARDs. For such patients, guidelines recommend the addition of either a 
biologic DMARD (bDMARD) or a targeted synthetic DMARD (tsDMARD) [3], which target specific molecules or molecular structures (targeted therapy). Typically, the first-choice targeted therapy is a tumor necrosis factor inhibitor (TNFi) [4].

Patients with RA who continue to experience disease symptoms fail to achieve disease remission or low disease activity [5], ultimately progress through different courses of treatment, either cycling to the same type of targeted therapy or switching to a targeted therapy with a different mode of action (MOA switching). Currently, limited data are available outside the US on targeted therapy prescribing patterns of rheumatologists and the reasons behind prescribing.

Our study aimed to obtain data on the prescribing patterns and reasons for current RA treatment practice in Europe and Japan.

\section{Ethics Approval}

The research was granted permission by the centralized Freiburger Ethik-Kommission (FEKI). FEKI code 017/1168. ID number: 175296617. Informed consent was required for both physicians and patients to take part in the study.

\section{METHODS}

\section{Data Source}

Data were extracted from the Adelphi Real World RA Disease Specific Programme ${ }^{\mathrm{TM}}$ (DSP) collected in Q1 and Q2 2017. DSPs are large, cross-sectional, point-in-time surveys conducted to provide observations of real-world clinical practice for many common chronic diseases. They collect data from key sources of information such as physician interviews, physician workload questionnaires, Patient Record Forms (PRF), and patient questionnaires $[6,7]$. The DSP methodology has been validated [6-8] and adapted as appropriate for market variations over 11 waves of the program (i.e., since information was first collected). The
Adelphi Real World RA DSP is a cross-sectional, single-point-in-time survey of qualified rheumatologists and their adult patients with RA in France, Germany, Italy, Spain, and the UK (EU5), and rheumatologists and orthopedists in Japan. Physicians were identified by publicly available lists and, a geographically diverse sample of physicians were recruited by fieldbased interviewers and were compensated according to fair market research rates.

\section{Survey}

Physicians completed surveys about their weekly workload and PRF for the next ten consecutive eligible RA patients they saw in their clinic (based on inclusion criteria below).

The PRF contained detailed questions on patient demographics, current treatment, reasons for targeted therapy choice, and general patient management (e.g., frequency of consultation with the treating and other physicians). To classify patient's disease severity, physicians were asked: "What was your overall assessment of the severity of RA in this patient based on your own definitions of the terms mild, moderate and severe immediately prior to initiation of the current treatment?". To determine if patients were at the maximum csDMARD dose physicians were asked: "Was the maximum recommended dose of the csDMARD reached before initiating the targeted therapy yes, no, or don't know?". It is important to note that the maximum dose of csDMARDs differs across countries (e.g., in Europe the maximum dose of methotrexate is $25 \mathrm{mg} /$ week; however, in Japan the maximum dose of methotrexate is $16 \mathrm{mg} /$ week). To determine if patients discontinued their csDMARD upon initiation of targeted therapy, physicians were asked: "Was the csDMARD discontinued upon initiation of targeted therapy - yes, no or don't know?".

All responses captured on the PRFs were anonymized. All participating physicians and patients were assigned a study number to allow linkage of data during the processing stage. 


\section{Inclusion and Exclusion Criteria}

\section{Patients}

Patients were included in this study if they were $\geq 18$ years old with a diagnosis of RA (no requirement to perform any diagnostic test or assessments), had a complete RA targeted therapy history, not currently involved in a clinical trial, and were visiting a participating physician.

\section{Physicians}

In EU5 and Japan, physicians who were seeing $\geq 10$ patients or $\geq 5$ patients, respectively, with RA per month were included.

\section{Analysis Definitions}

Patients with complete RA targeted therapy (bDMARD or tsDMARD) history were analyzed in this study.

\section{First-and Second-Line Targeted Therapy}

Patients were grouped based on the first-line targeted therapy that they received, either a TNFi (etanercept, adalimumab, infliximab, certolizumab pegol, golimumab) or non-TNFi (abatacept, rituximab, anakinra, and tocilizumab) or tofacitinib (Japan only, as tofacitinib was approved but not available in Europe at the time of data collection). Second-line therapy was defined as a targeted therapy (TNFi, nonTNFi or tofacitinib) that was prescribed to patients following first-line targeted therapy failure as either monotherapy or combination therapy.

\section{Monotherapy and Combination Therapy}

Monotherapy was defined as a prescription for a targeted therapy without a concomitant prescription for a csDMARD. Combination therapy was defined as a prescription for a targeted therapy with a concomitant prescription for any csDMARD (e.g., methotrexate, leflunomide).

\section{TNFi Cycling and MOA Switching}

TNFi cycling was defined as a prescription for a TNFi as both first- and second-line targeted therapy. MOA switching was defined as a prescription for a TNFi as first-line targeted therapy and a non-TNFi as second-line targeted therapy. For the TNFi cycling analysis, patients were excluded if they initiated treatment prior to June 2006, this was to allow availability of nonTNFi into the market (which were first introduced and widely available across the world in 2006).

\section{Statistical Analysis}

Numerical variables were analyzed using mean, standard deviation (SD), median, and minimum and maximum values, and categorical variables were analyzed by frequency and percentage of patients falling into each category. Univariate analysis compared the patient groups based on physician responses about treatment and patient characteristics. When there were two comparison groups, Mann-Whitney tests were used for numeric variables, and Fisher's exact tests were used for binary categorical variables. When there were $\geq 3$ comparison groups, Kruskal-Wallis tests were used for numeric variables, and Chi-squared tests were used for categorical variables with more than two groups. Survival estimates for time to discontinuation of first-line targeted therapy were calculated using the Kaplan-Meier method. Differences between first-line targeted therapy class were assessed using log-rank tests. Where statistical tests were performed, $P$ values $<0.05$ were considered statistically significant. All analyses were performed by Adelphi Real World using Stata 15.0 or later (StataCorp, Stat Statistical Software: Release 15. College Station, TX: StataCorp LP). 


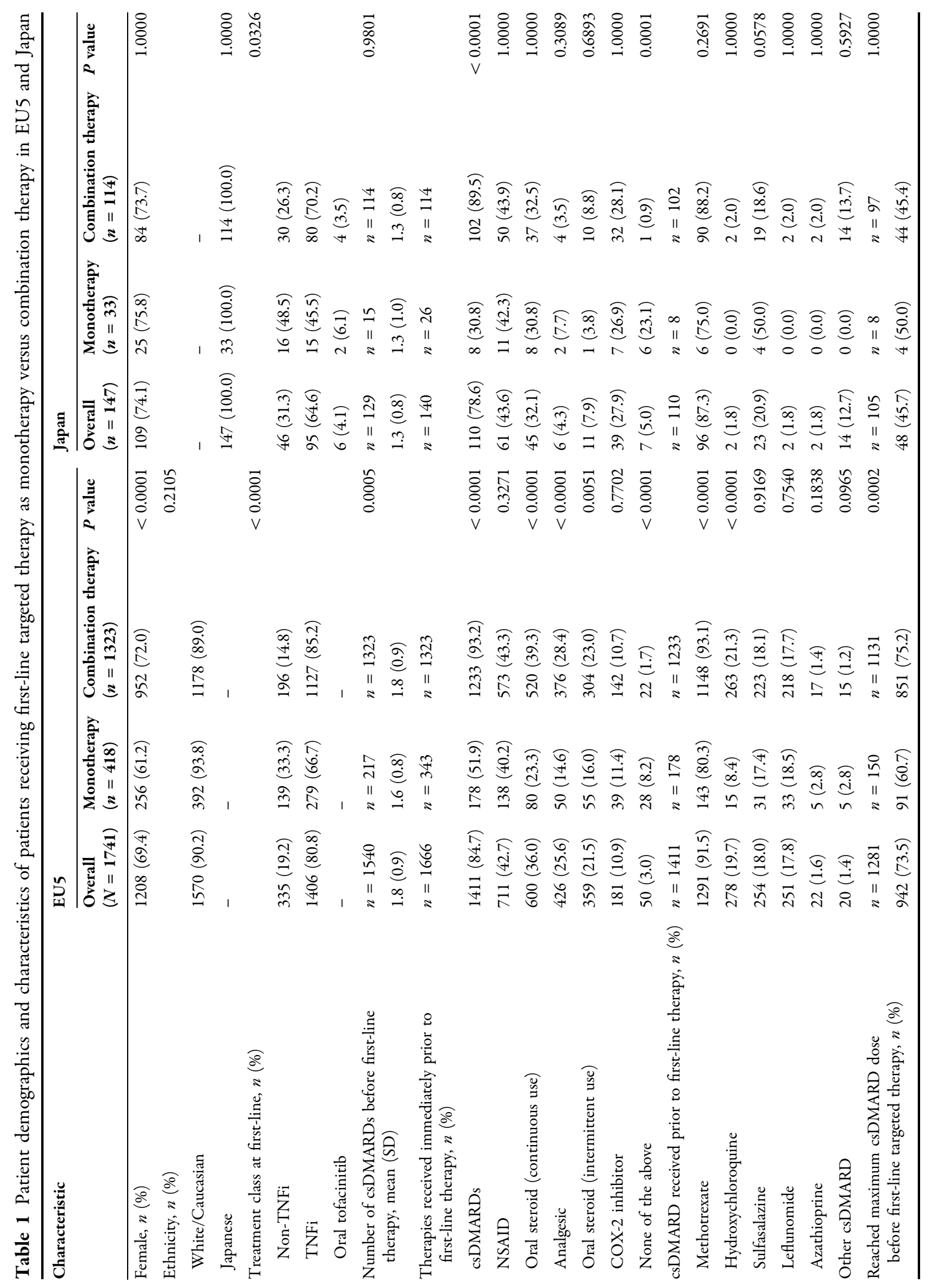




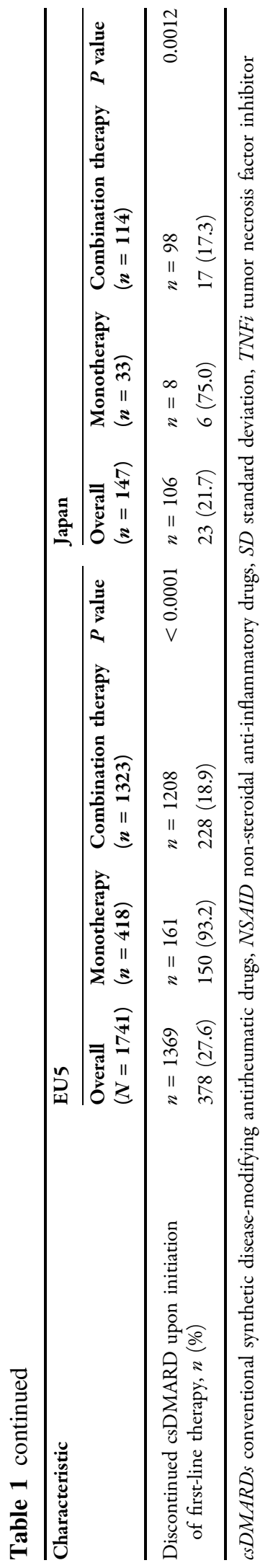

\section{RESULTS}

\section{First-Line Monotherapy or Combination Therapy Targeted Treatment Use}

In EU5, the overall DSP sample included 3088 patients from 301 rheumatologists. Of these, the first targeted therapy after 2006 could be identified for 1741 patients who were included in this study, and $76.0 \%$ of patients were prescribed combination therapy (Table 1 and Supplementary Fig. 1A). In Japan, the overall DSP sample included 347 patients from 42 physicians ( 11 rheumatologists, 31 orthopedists). Of these, the first targeted therapy could be identified for 147 patients who were included in this study and $77.6 \%$ of patients were prescribed combination therapy (Table 1 and Supplementary Fig. 1B). Patient demographics and characteristics for monotherapy and combination patients are shown in Table 1.

In both EU5 and Japan, csDMARDs were the most common therapy received immediately prior to first-line targeted therapy $(84.7 \%$ and $78.6 \%$, respectively) and this was most commonly methotrexate $(91.5 \%$ and $87.3 \%$, respectively). NSAIDs were the second most common therapy received immediately prior to first-line targeted therapy $(42.7 \%$ and $43.6 \%$, respectively). In EU5, a smaller proportion of monotherapy patients were prescribed methotrexate or hydroxychloroquine immediately prior to targeted therapy compared with combination therapy patients (both $P<0.05$; Table 1). Other therapies received immediately prior to first-line targeted therapy included nonsteroidal anti-inflammatory drugs (NSAIDs), oral steroids, analgesics, and COX-2 inhibitors (Table 1).

In EU5, $73.5 \%$ of patients reached the maximum csDMARD dose as reported by their physician before initiating first-line targeted therapy, with a greater proportion of combination therapy patients reaching the maximum dose before first-line targeted therapy than monotherapy patients $(P<0.05)$. While in Japan, $45.7 \%$ of patients reached the maximum csDMARD dose before first-line targeted therapy 
and this was similar between the monotherapy and combination therapy groups (Table 1).

Median time to discontinuation of first-line targeted therapy was significantly different between patients receiving first-line monotherapy versus combination therapy in EU5 $(P<0.001)$ but not in Japan $(P=0.25)$ (Fig. 1a, b).

\section{Targeted Therapy at First-Line}

At initiation of first-line targeted therapy, 1406 (80.8\%) patients in EU5 were prescribed a TNFi and $335(19.2 \%)$ were prescribed a non-TNFi. No patients were prescribed tsDMARD in EU5 as the only available product, tofacitinib, while approved, was not available at the time of data collection (Table 2 and Supplementary Fig. 1A). In Japan, 95 patients $(64.6 \%)$ were prescribed a TNFi as first-line targeted therapy, 46 (31.3\%) were prescribed a non-TNFi, and six were prescribed a tsDMARD (4.1\%) (Table 2 and Supplementary Fig. 1B). Patient demographics and characteristics for all patients are shown in Table 2.

In EU5, $48.3 \%$ of patients had physicianreported moderate disease and $45.6 \%$ had severe disease at initiation of first-line targeted therapy, while in Japan, $62.3 \%$ of patients had physician-reported moderate disease and $18.5 \%$ had severe disease. Disease severity was different between patients initiating a non-TNFi versus a TNFi $(P<0.05)$ in EU5 but was not different between the treatment groups in Japan (Table 2).

In EU5 and Japan, csDMARDs were the most commonly used therapy immediately prior to first-line targeted therapy followed by NSAIDs (treatments are not mutually exclusive and could be used in combination or as monotherapy, data not collected). The most commonly prescribed first-line targeted therapy was etanercept in EU5 (33.9\%), and tocilizumab in Japan (22.4\%; Table 2). Physicians' reasons for targeted therapy choice were known for 1322 patients in EU5 and 119 patients in Japan. In EU5 and Japan, physician reasoning that varied significantly between non-TNFi and TNFi use is presented in Supplementary Table 1. The top three reasons physicians in EU5 $(n=1322)$ gave for prescribing each specific type of first-line targeted therapy were 'strong overall efficacy' (75.3\%), 'inhibition of disease progression' $(46.4 \%)$, and 'good overall safety profile' $(40.0 \%)$. In Japan $(n=119)$, the top three reasons were 'strong overall efficacy' (77.3\%), 'fast onset of action' (53.8\%), and 'inhibition of disease progression' (55.5\%) (Fig. 2a, b).

Median time to discontinuation of first-line targeted therapy was significantly different between the treatment classes received in EU5 $(P=0.0002)$ but was not significantly different in Japan $(P=0.3991)$ (Fig. 1c, d).

\section{TNFi Cycling Versus MOA Switching at Second-Line Targeted Therapy}

In EU5, 418 patients progressed to second-line targeted therapy and of these, 38 patients received monotherapy. After excluding patients who initiated therapy prior to 2006, 365 patients who received TNFi as first-line targeted therapy progressed to second-line targeted therapy; $47.9 \%$ were TNFi cyclers and $52.1 \%$ were MOA switchers (Table 3 and Supplementary Fig. 1A). In Japan, 22 patients who received TNFi as first-line targeted therapy progressed to second-line targeted therapy; $45.5 \%$ were TNFi cyclers and $54.5 \%$ were MOA switchers (Table 3 and Supplementary Fig. 1A). Due to the small number of patients $(n=22)$, results from Japan are not described in detail but are summarized in Table 3. Patient demographics and characteristics are also shown in Table 3.

Time from diagnosis to second-line targeted therapy initiation was 7.4 (7.6) years for patients in EU5; TNFi cycling patients had a longer time from diagnosis to second-line targeted therapy initiation than MOA switchers $(P=0.04)$.

In EU5, at initiation of second-line targeted therapy, physician-reported disease severity was significantly worse in MOA switching patients than TNFi cycling patients $(P<0.05$; Table 3$)$. Almost half of patients in EU5 had moderate RA when second-line targeted therapy was initiated (49.9\%; Table 3). Among TNFi cycling patients, 

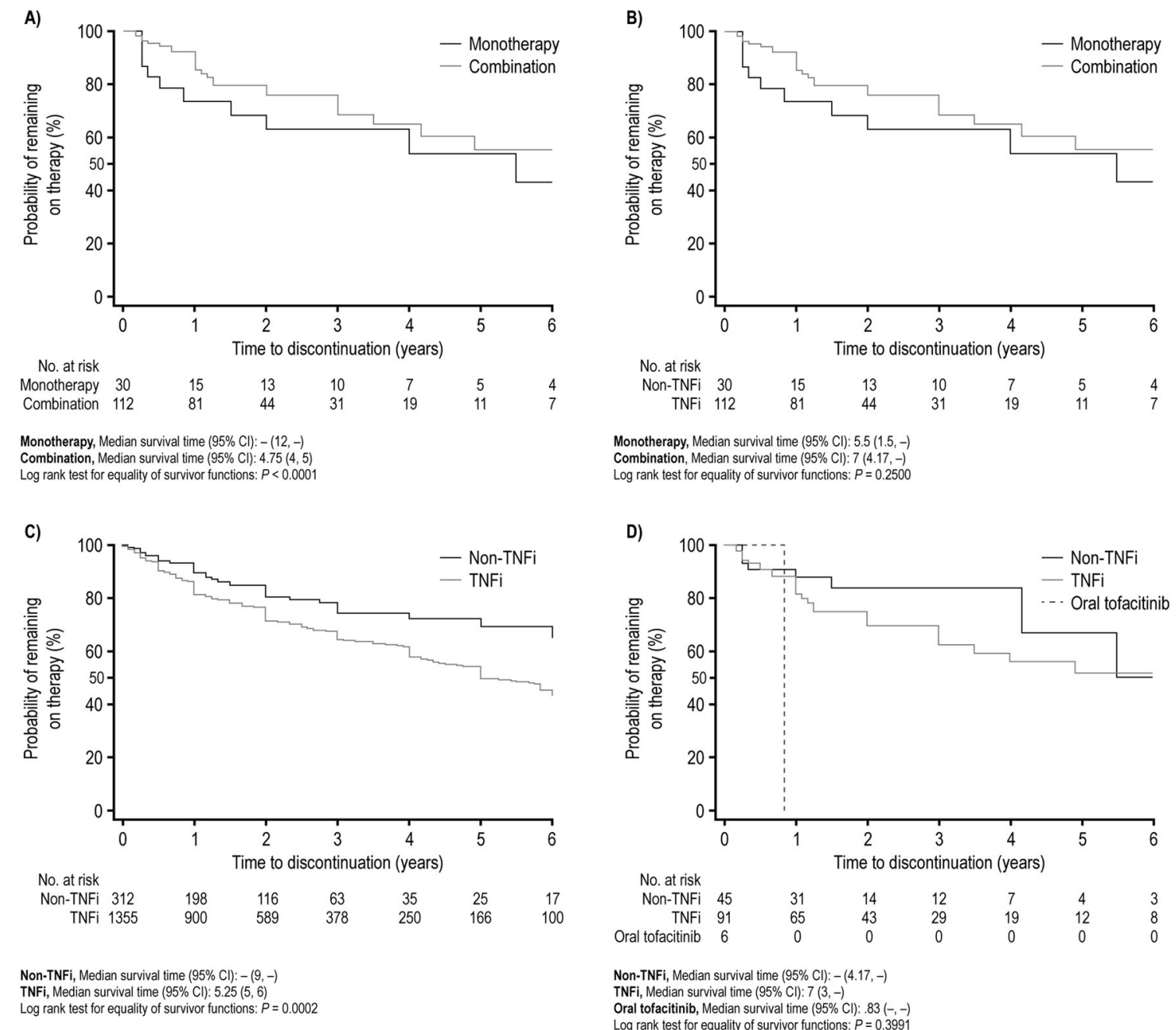

Fig. 1 Kaplan-Meier survival estimates for time to discontinuation of first-line targeted therapy for a monotherapy versus combination therapy in EU5;

the most frequently prescribed targeted therapy in EU5 was etanercept (34.9\%), while tocilizumab was the most frequently prescribed targeted therapy among MOA switchers (37.9\%; Table 3).

Physicians' reasons for moving patients from their first-line to their second-line targeted therapy were known for 316 patients in EU5, and the top reason given was 'secondary lack of efficacy' (46.2\%; Fig. 3a). In EU5, physician reasoning that varied significantly between TNFi cycling and MOA switching patients were: 'worsening condition', 'patient required a targeted therapy with a different MOA', 'formulary

b Monotherapy versus combination therapy in Japan; c TNFi versus non-TNFi in EU5; d TNFi versus nonTNFi in Japan

driven switch' (Fig. 3a), and 'comorbidities' $(P<0.05$; data not shown). Physician reasoning data for patients in Japan are not described due to the small number of patients in this analysis $(n=13)$ but are presented in Fig. 3b.

\section{DISCUSSION}

With the number of treatment options increasing for patients with RA, there is a growing need to understand prescribing patterns and behaviors to help optimize treatment outcomes. As limited data are available outside the US on 


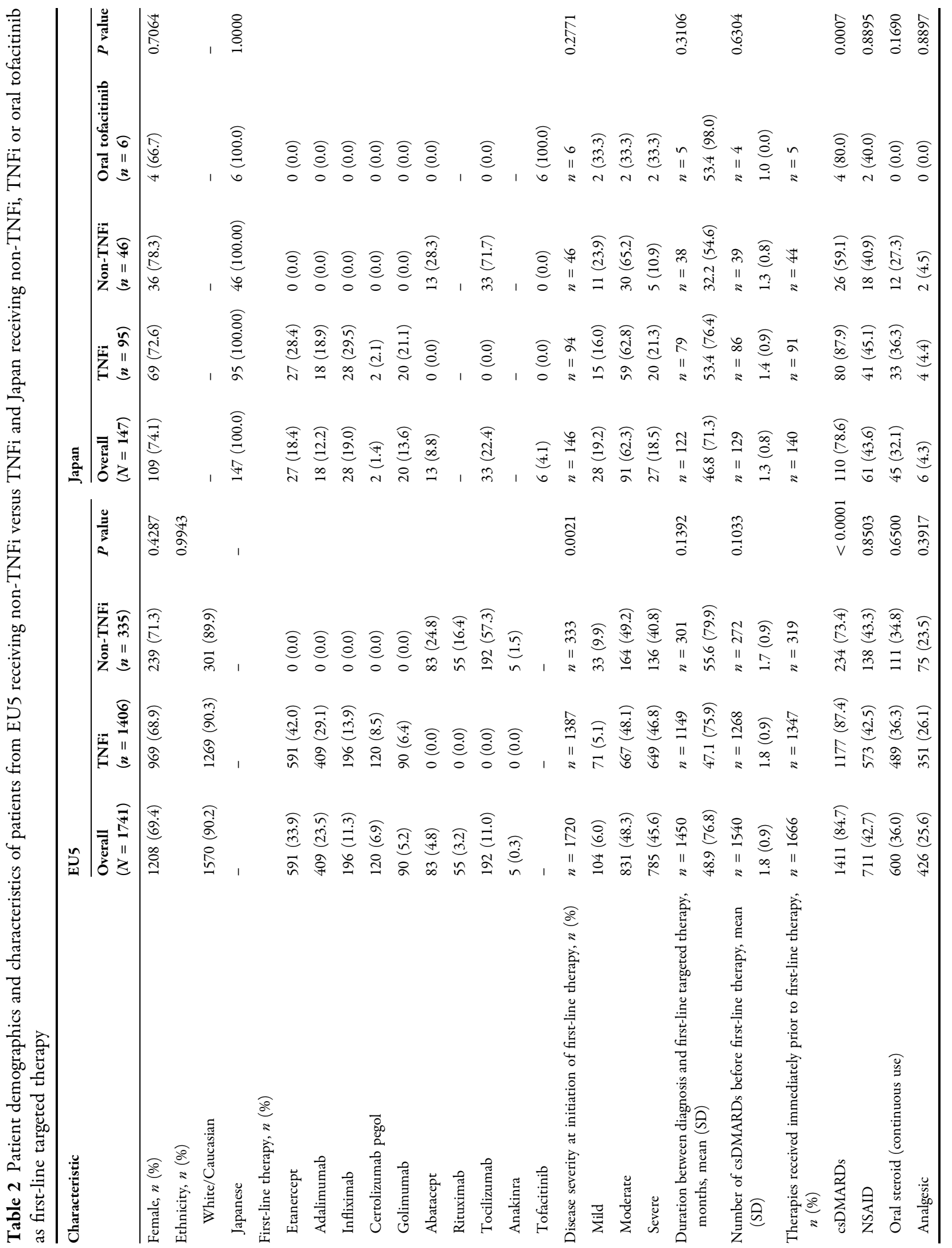




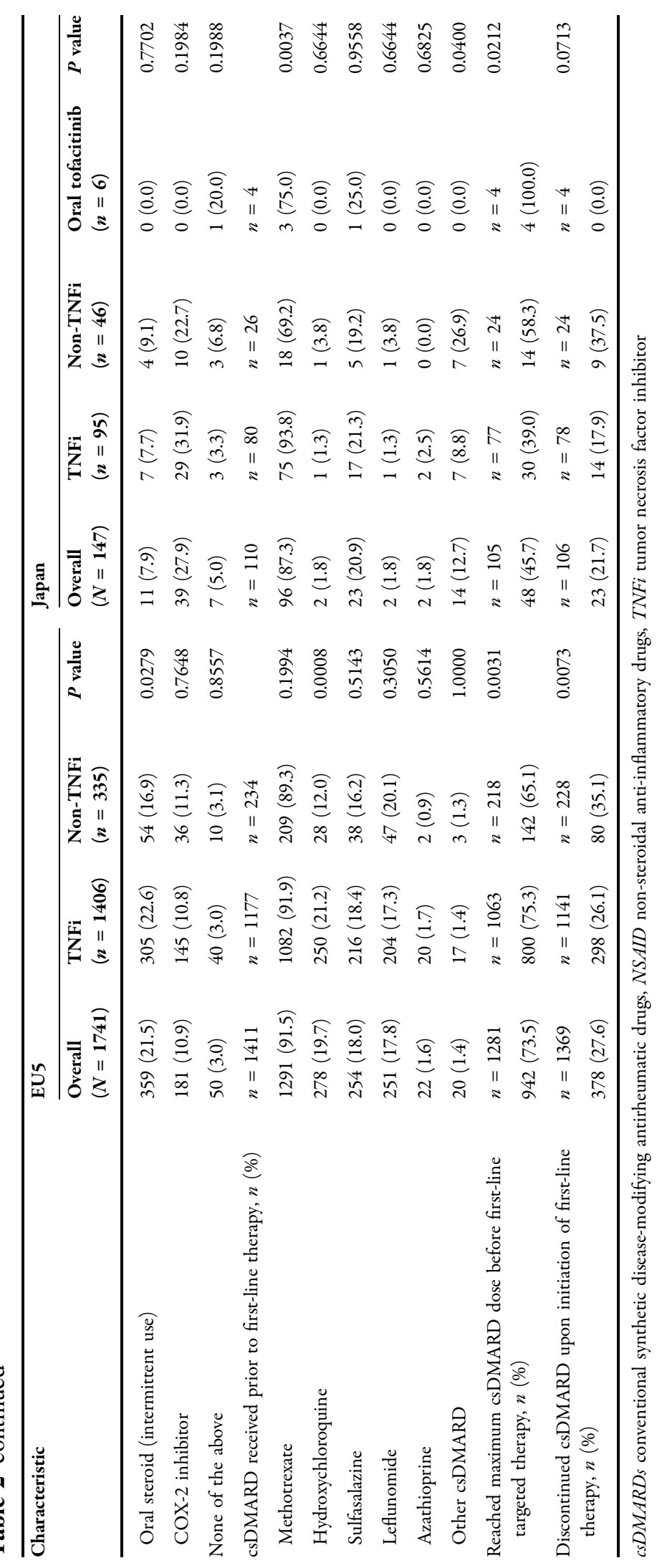




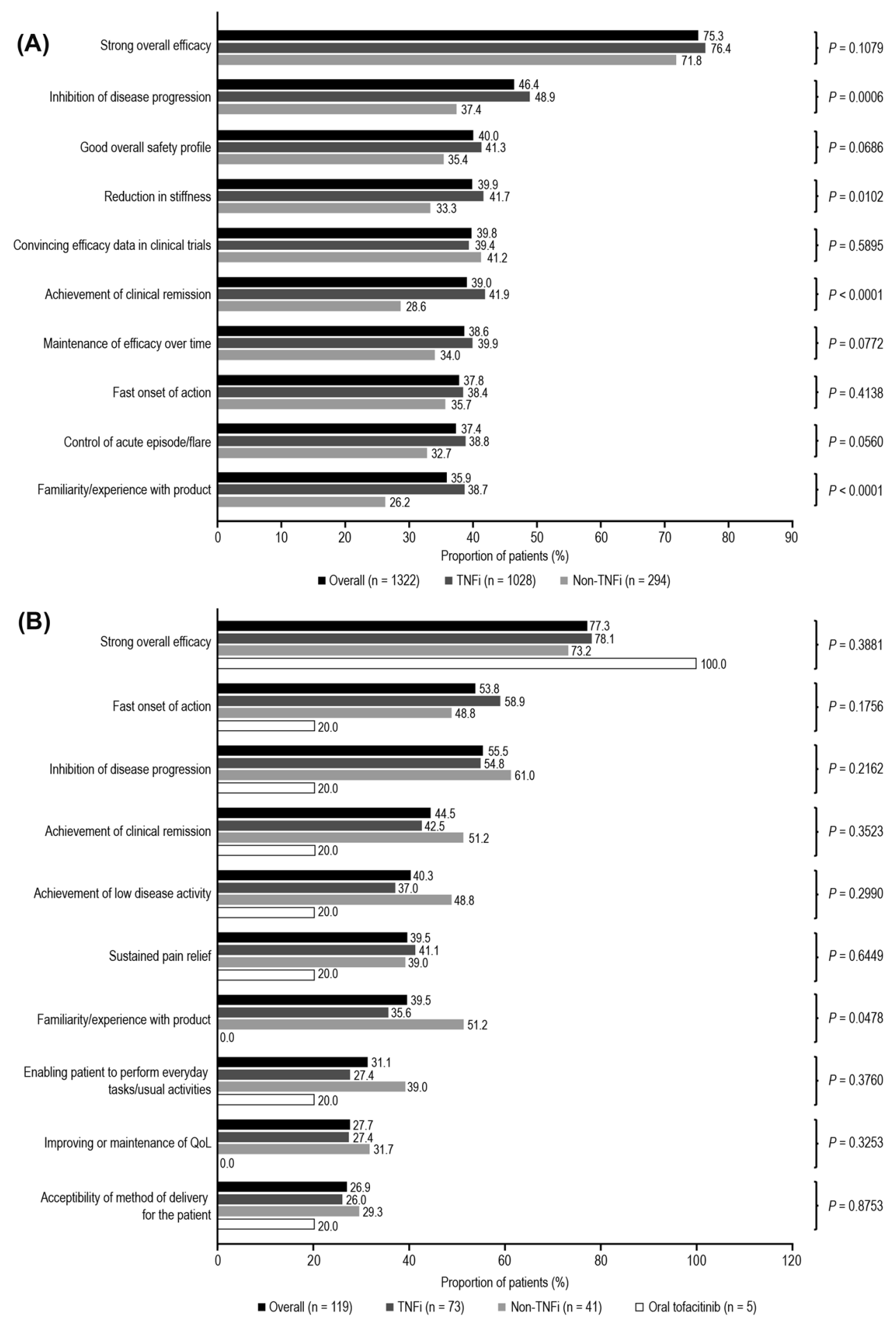

Fig. 2 Top 10 reasons for physicians choosing the current therapy as a first-line targeted therapy in a EU5 and; b Japan. QoL quality of life, $T N F i$ tumor necrosis factor inhibitor

targeted therapy behaviors that influence prescribing patterns, the current study surveyed rheumatologists and orthopedists to provide a subjective perspective across EU5 and Japan. 


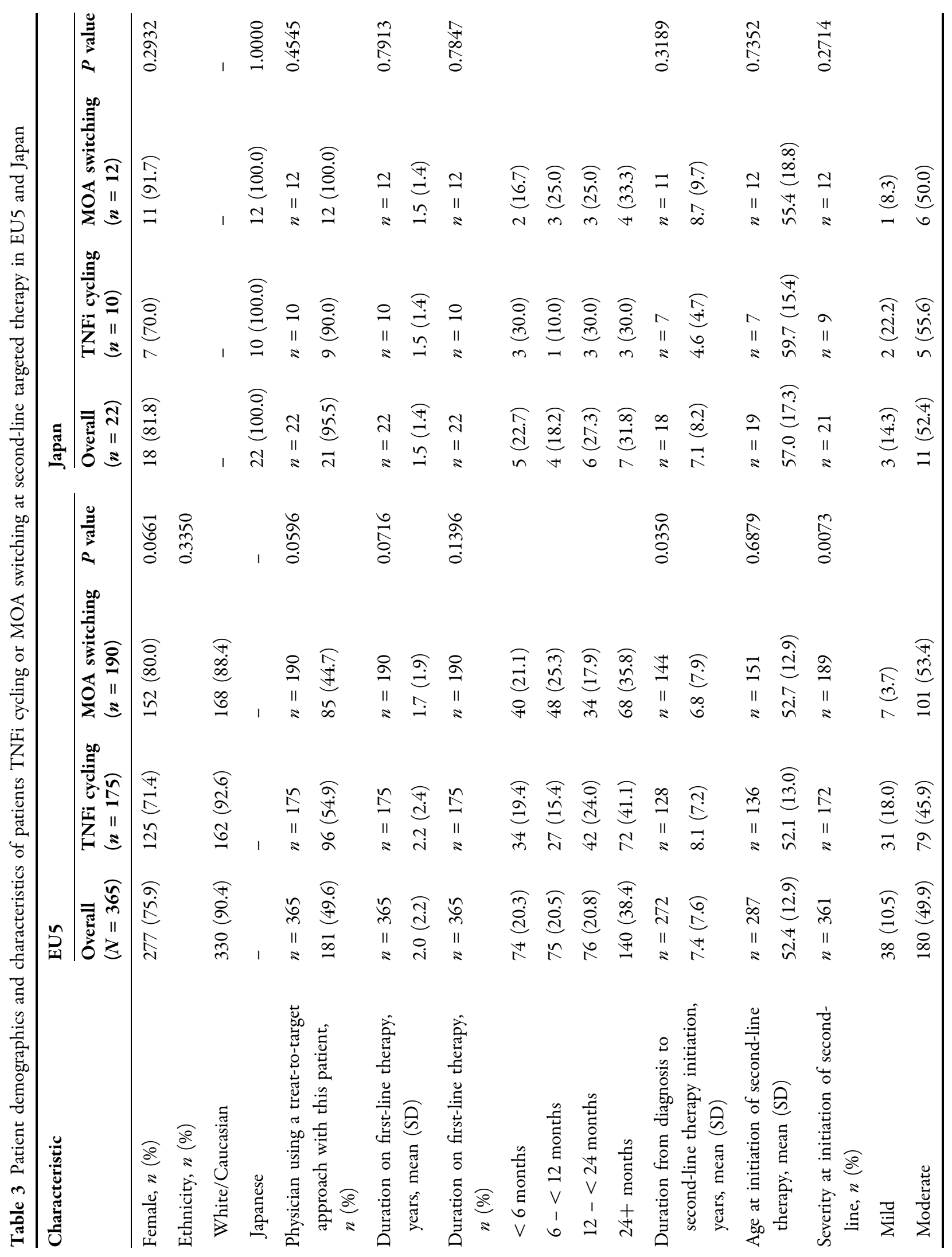




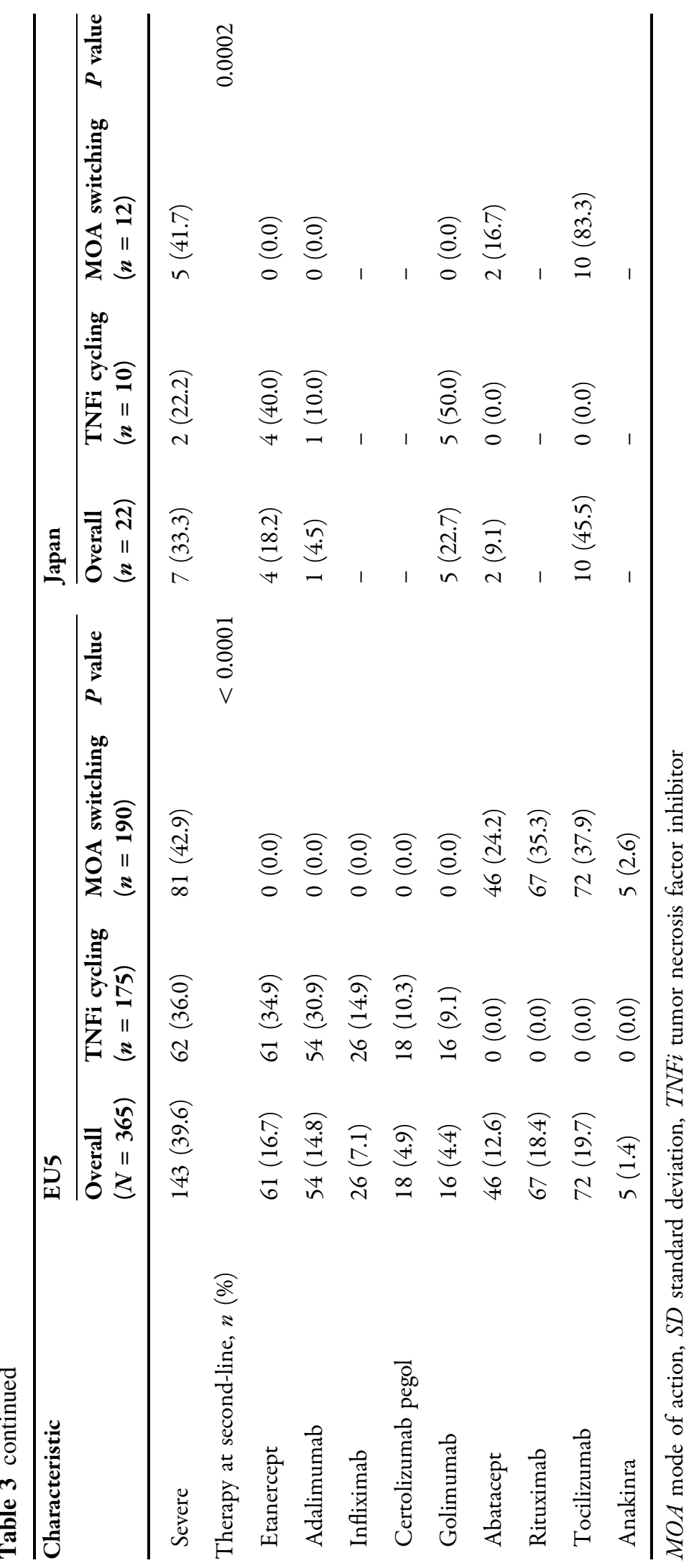




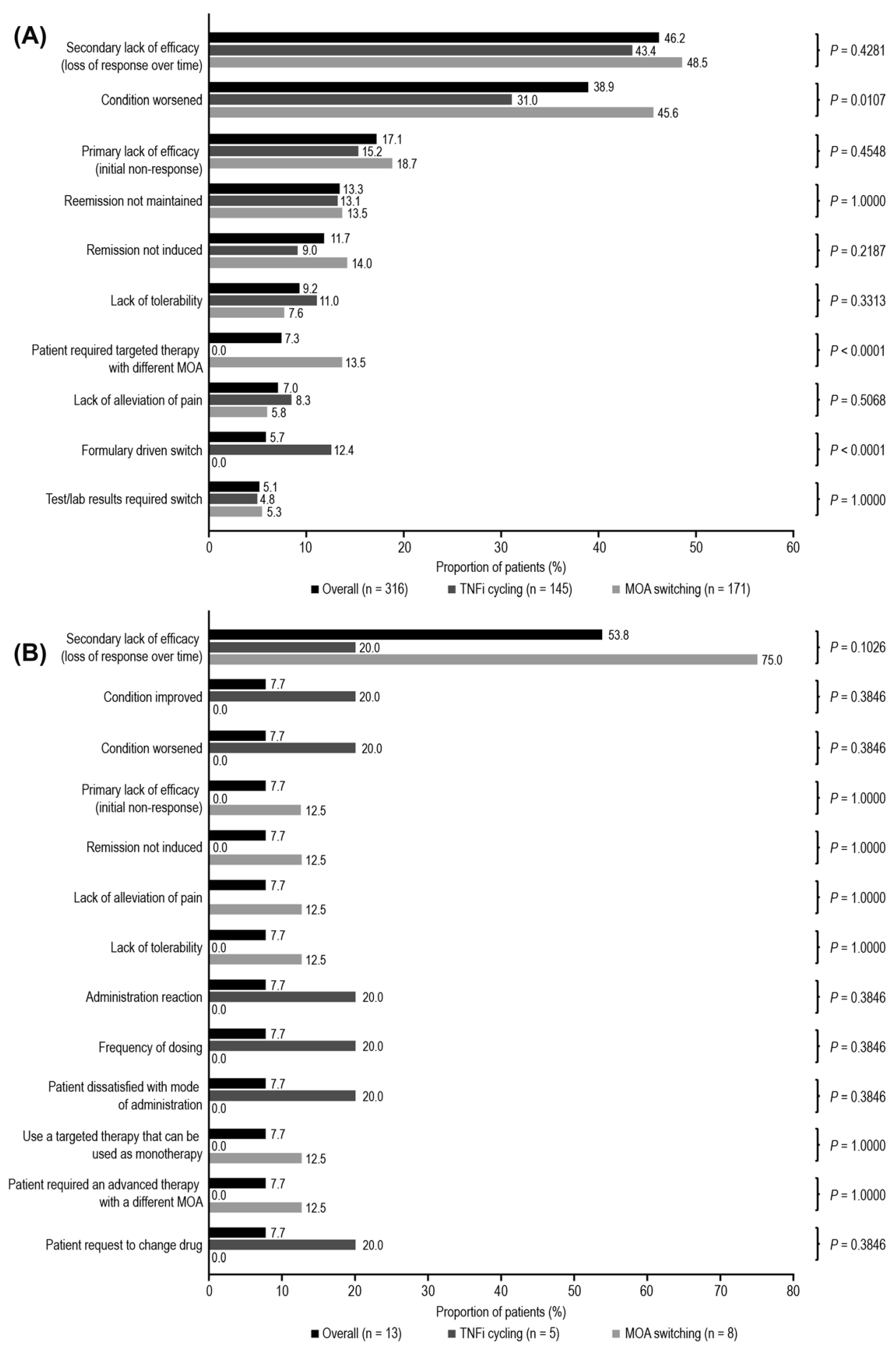

Fig. 3 Top reasons physicians gave for switching therapy following initial first-line targeted therapy in a EU5 and; $\mathbf{b}$ Japan. $M O A$ mode of action, TNFi tumor necrosis factor inhibitor

Based on results from clinical studies [9], treatment guidelines recommend that moderate-to-severe RA patients who have inadequate disease control or fail to respond to csDMARD monotherapy, could potentially initiate a targeted therapy combined with a csDMARD [3]. 
The results of this current study suggest that physicians are largely acting in alignment with current treatment guidelines and available data, as more than three quarters of patients in EU5 and Japan were prescribed combination therapy. Our results are slightly higher than those previously observed in other countries, including the US, where studies have demonstrated that $55-75 \%$ of RA patients utilized combination therapy [10-14]. Monotherapy use in around one-quarter of patients in the current study could be related to patient belief regarding the safety of some csDMARDs, such as methotrexate, which would lead to non-adherence and reluctance to use csDMARDs [15]. It should be noted though that targeted monotherapy use may be underestimated in the current study due to the prevalence of patient non-adherence to combination therapy, which was not investigated. It should be noted that although most physicians largely act in alignment with the current treatment guidelines, in the current study around half (51\%) of first-line monotherapy patients from EU5 utilized a csDMARD prior to initiating first-line targeted therapy. This could have been due to csDMARD intolerance, and as the European label states that etanercept is acceptable as monotherapy in patients who do not tolerate methotrexate, it may explain why etanercept was prescribed more commonly (33.9\% prescribed at first-line) [16]. It is also interesting to note that the number of patients who reached the maximum csDMARD dose before initiating first-line targeted therapy was higher in EU5 than Japan (73.5 vs. $45.7 \%)$. In Europe, treatment guidelines recommend a methotrexate dosage up to $25 \mathrm{mg} /$ week [3], while in Japan, methotrexate is approved at a dosage of $16 \mathrm{mg} /$ week; however, more than half of Japanese patients with RA are unable to tolerate a dose of $16 \mathrm{mg} /$ week [17]. The maximum dose of csDMARDs that is approved/can be tolerated by patients in EU5 and Japan may be explained by body weight and body mass index, which are considerably lower in Japanese patients than in patients from EU5 $[18,19]$.

The current study also found that many patients in EU5 and Japan were prescribed a TNFi as first-line targeted therapy $(80.8 \%$ and
64.6\%, respectively). Although the European guidelines do not specifically state that a TNFi should be used as a first-line targeted therapy, typically the first-choice targeted therapy is a TNFi [4]. Examining prescription behaviors in RA treatment revealed that Japanese physicians were more likely to prescribe tocilizumab as the first-line targeted therapy (22\%), while physicians in EU5 did not prescribe tocilizumab to the same extent (11\%). The higher prescription rates for tocilizumab may be because it was developed in Japan and was the first interleukin-6 receptor inhibitor approved for RA treatment in Japan [20]. Previous studies have suggested that tocilizumab may be more costeffective than other bDMARDs including adalimumab and etanercept [21, 22], while in a 5-year study, tocilizumab monotherapy demonstrated sustained long-term efficacy [23], which may also explain why physicians in Japan favored tocilizumab as the first-line therapy. It may also be possible that the differences observed in treatment patterns could be due to differences in healthcare across the world that may be more notable between EU5 and Japan. It is also interesting to note that in the current study, $5-10 \%$ of patients in the EU5 that were prescribed a targeted therapy had characteristics of mild RA, despite European labeling for targeted therapies recommending use in patients with moderate-to-severe RA. Physicians classified a patient's disease severity in the current study based on their own definitions of the terms mild, moderate, and severe, and not on common scores such as disease activity score at 28 joints (DAS-28); therefore, it may have been possible that a patient classified as mild would have actually been moderate or severe based on validated measures.

Following first-line targeted therapy failure, patients generally fall into one of two groups, TNFi cyclers or MOA switchers [4]. The current study found that in EU5 and Japan, just over half of patients were MOA switchers. These results differ from earlier studies conducted in the US and the UK alone between 2005 and 2013, which revealed that more patients in clinical practice cycle between TNFi rather than switch to a targeted therapy with a different MOA [24-26]. Nonetheless, despite multiple 
studies demonstrating the benefits of switching to a targeted therapy with a different MOA, including higher treatment persistence [27-29], a greater reduction in disease activity $[29,30]$, lower RA-related drug and medical care costs [28], decreased likelihood of failure [31], and increased likelihood of physical function improvement $[25,32,33]$ higher rates of TNFi cycling were observed. Although guidelines and multiple studies have suggested the switch to a targeted therapy with a different MOA following first-line targeted therapy failure, it is not compulsory, and the trends observed in the current study including a high rate of TNFi cycling may be dependent on factors such as primary versus secondary failure to TNFi or antidrug-antibodies. This study is potentially the first to investigate physician rationale around prescribing targeted therapy in EU5 and Japan. A previous study conducted with US physicians examined treatment patterns for a similar set of RA therapies and found that the variability may be explained by patient and physician preference, physician experience and specialty, and medication cost [34]. The top reasons these US physicians gave for prescribing each specific type of first-line targeted therapy were similar between the European and Japanese physicians. In EU5, these were strong overall efficacy, inhibition of disease progression, and overall good safety profile, and in Japan, these were strong overall efficacy, fast onset of action, and inhibition of disease progression. These results confirm that efficacy and disease control are two of the most important factors physicians consider when prescribing treatment for moderate-to-severe RA. Other retrospective cohort studies have examined the reasons for MOA switching or discontinuing targeted therapy have found that lack of efficacy and adverse events were the main reasons physicians gave for changing treatment [35]. In this current study, the top reason physicians gave for switching following initial first-line targeted therapy in EU5 and Japan was secondary lack of efficacy. It is important to note that patient reasoning for targeted therapy decisions was not examined in the current study. As the healthcare system now favors increased patient participation during the treatment decision- making process and due to the large number of targeted therapy options available for patients with RA [36], it may be interesting to determine how patient reasoning fits into targeted therapy decisions in future studies.

\section{Strength and Limitations}

The strengths of this study include recruitment of a geographically diverse sample of physicians and patients across EU5 and Japan, capturing patients from physician practices, and standardized data collection tools. However, it is important to note some limitations of the current study. Firstly, physicians were asked to provide data for a consecutive series of patients to avoid selection bias, but no formal patient selection verification procedures were in place. Secondly, while the identification of the target patient group was based on judgment of the responding physician and not a formalized diagnostic checklist, it was representative of the real-world classification of the patient. Furthermore, classification of disease activity/disease severity of RA patients was not based on DAS-28 and could therefore be subjective and variable. Also, the diagnosis of RA is subjective and depends on the individual rheumatologist, which is another limitation of the study, as the number of patients with RA could have been under- or over-estimated. It should also be noted that inclusion of orthopedists in Japan is a limitation of the physician sample. Additionally, the percentage of patients utilizing monotherapy may be underestimated due to potential nonadherence of the csDMARD, which was not examined in the study. Finally, the small number of patients receiving tofacitinib in Japan should be noted, as this could affect interpretation of the results.

\section{CONCLUSIONS}

Most patients in the current study were prescribed combination therapy and most received a TNFi at first-line. The main reason physicians gave for changing targeted therapy was secondary lack of efficacy. Despite the demonstrated benefits associated with MOA switching, 
almost half of patients were cycled to another TNFi. These results suggest that physicians in EU5 and Japan generally follow treatment guidelines but prescribe how they choose, providing optimized and individualized treatments for their patients.

\section{ACKNOWLEDGEMENTS}

Funding. This study and the journal's Rapid Service Fee were funded by Sanofi and Regeneron Pharmaceuticals, Inc.

Medical Writing Assistance. Medical writing support provided by Abby Armitt, MSc, Prime, Knutsford, Cheshire, UK, which was funded by Sanofi and Regeneron Pharmaceuticals, Inc.

Authorship. All named authors meet the International Committee of Medical Journal Editors (ICMJE) criteria for authorship for this article, take responsibility for the integrity of the work as a whole, and have given their approval for this version to be published.

Disclosures. Emma Sullivan is a former employee of Adelphi Real World, a company that received research funding from Sanofi and Regeneron Pharmaceuticals, Inc. Emma Sullivan is now an employee of Adelphi Values, Bollington, UK. Jim Kershaw and Stuart Blackburn are employees of Adelphi Real World, a company that received research funding from Sanofi and Regeneron Pharmaceuticals, Inc., for the current study. Puneet Mahajan is an employee of and stockholder in Sanofi. Susan Boklage is a former employee of and stockholder in Regeneron Pharmaceuticals, Inc. Susan Boklage is now an employee of GSK, Collegeville, PA, USA.

Compliance with Ethics Guidelines. The research was granted permission by the centralized Freiburger Ethik-Kommission (FEKI). FEKI code 017/1168. ID number: 175296617. Informed consent was required for both physicians and patients to take part in the study.
Data Availability. Data are not publicly available; however, would be available on reasonable request to external parties, after request review by Adelphi Real World executive committee.

Open Access. This article is licensed under a Creative Commons Attribution-NonCommercial 4.0 International License, which permits any non-commercial use, sharing, adaptation, distribution and reproduction in any medium or format, as long as you give appropriate credit to the original author(s) and the source, provide a link to the Creative Commons licence, and indicate if changes were made. The images or other third party material in this article are included in the article's Creative Commons licence, unless indicated otherwise in a credit line to the material. If material is not included in the article's Creative Commons licence and your intended use is not permitted by statutory regulation or exceeds the permitted use, you will need to obtain permission directly from the copyright holder. To view a copy of this licence, visit http:// creativecommons.org/licenses/by-nc/4.0/.

\section{REFERENCES}

1. Gibofsky A. Epidemiology, pathophysiology, and diagnosis of rheumatoid arthritis: a synopsis. Am J Manag Care. 2014;20(7 Suppl):S128-S135135.

2. Aletaha D, Neogi T, Silman AJ, Funovits J, Felson DT, Bingham CO 3rd, et al. 2010 Rheumatoid arthritis classification criteria: an American College of Rheumatology/European League Against Rheumatism collaborative initiative. Arthritis Rheum. 2010;62(9):2569-81.

3. Smolen JS, Landewé R, Bijlsma J, Burmester G, Chatzidionysiou $\mathrm{K}$, Dougados $\mathrm{M}$, et al. EULAR recommendations for the management of rheumatoid arthritis with synthetic and biological diseasemodifying antirheumatic drugs: 2016 update. Ann Rheum Dis. 2017;76(6):960-77.

4. Moots RJ, Naisbett-Groet B. The efficacy of biologic agents in patients with rheumatoid arthritis and an inadequate response to tumour necrosis factor inhibitors: a systematic review. Rheumatology (Oxford). 2012;51(12):2252-61. 
5. Hamann PDH, Pauling JD, McHugh N, Shaddick G, Hyrich K, BSRBR-RA Contributors Group. Predictors, demographics and frequency of sustained remission and low disease activity in anti-tumour necrosis factor-treated rheumatoid arthritis patients. Rheumatology (Oxford). 2019;58(12): 2162-69.

6. Babineaux SM, Curtis B, Holbrook T, Milligan G, Piercy J. Evidence for validity of a national physician and patient-reported, cross-sectional survey in China and UK: the Disease Specific Programme. BMJ Open. 2016;6(8):e010352.

7. Anderson P, Benford M, Harris N, Karavali M, Piercy J. Real-world physician and patient behaviour across countries: disease-specific programmes-a means to understand. Curr Med Res Opin. 2008;24(11):3063-72.

8. Higgins V, Piercy J, Roughley A, Milligan G, Leith A, Siddall J, et al. Trends in medication use in patients with type 2 diabetes mellitus: a long-term view of real-world treatment between 2000 and 2015. Diabetes Metab Syndr Obes. 2016;9:371-80.

9. Gómez-Reino J. Biologic monotherapy as initial treatment in patients with early rheumatoid arthritis. Rheumatology (Oxford). 2012;51(Suppl 5):v31-7.

10. Catay E, Bravo M, Rosa J, Soriano ER. Prevalence of biologics monotherapy in a cohort of patients with rheumatoid arthritis in daily clinical practice. BMC Musculoskelet Disord. 2016;17:110.

11. Emery P, Sebba A, Huizinga TWJ. Biologic and oral disease-modifying antirheumatic drug monotherapy in rheumatoid arthritis. Ann Rheum Dis. 2013;72(12):1897-904.

12. Choquette D, Thomas O, Arundine M. Lower than expected levels of DMARD acquisition immediately pre and post biologic initiation in rheumatoid arthritis patients [abstract number: 1841]. ACR/ ARHP annual meeting; 2012; Washington, DC.

13. Engel-Nitz NM, Ogale S, Kulakodlu M. Use of antitumor necrosis factor therapy: a retrospective study of monotherapy and adherence to combination therapy with non-biologic disease-modifying antirheumatic drugs. Rheumatol Ther. 2015;2(2): 127-39.

14. Pappas DA, Reed GW, Saunders K, John A, Shewade A, Greenberg JD, et al. Characteristics associated with biologic monotherapy use in biologic-naive patients with rheumatoid arthritis in a US registry population. Rheumatol Ther. 2015;2(1):85-96.

15. Leonardo $\mathrm{N}$, Lester, $\mathrm{S}$, Graham $\mathrm{M}$, Whittle, $\mathrm{S}$, Rowett D, Buchbinder R, Hill C. Attitudes and beliefs regarding methotrexate in patients with rheumatoid arthritis: results from Australian Rheumatology Association database [abstract number: 2353]. Arthritis Rheumatol. 2018;70(suppl 9). https://onlinelibrary.wiley.com/doi/epdf/10. 1002/art.40700.

16. European Medicines Agency. Enbrel Summary of Product Characteristics. 2009. https://www.ema. europa.eu/en/documents/product-information/enb rel-epar-product-information_en.pdf. Accessed 13 Mar 2020.

17. Kameda H, Fujii T, Nakajima A, Koike R, Sagawa A, Kanbe K, et al. Japan College of Rheumatology guideline for the use of methotrexate in patients with rheumatoid arthritis. Mod Rheumatol. 2019;29(1):31-40.

18. World Health Organization. Global Status Report on Noncommunicable Diseases 2014. 2014. https:// apps.who.int/iris/bitstream/handle/10665/148114/ 9789241564854_eng.pdf?sequence $=1$. Accessed 16 Mar 2020.

19. Miyasaka N. Treatment trends of rheumatoid arthritis in Japan: changes toward globalization and its unique innovation. Inflamm Regener. 2011;31: 25-32.

20. Sakai R, Cho S-K, Nanki T, Watanabe K, Yamazaki $\mathrm{H}$, Tanaka M, et al. Head-to-head comparison of the safety of tocilizumab and tumor necrosis factor inhibitors in rheumatoid arthritis patients (RA) in clinical practice: results from the registry of Japanese RA patients on biologics for long-term safety (REAL) registry. Arthritis Res Ther. 2015;17(1):74.

21. Carlson JJ, Ogale S, Dejonckheere F, Sullivan SD. Economic evaluation of tocilizumab monotherapy compared to adalimumab monotherapy in the treatment of severe active rheumatoid arthritis. Value Health. 2015;18(2):173-9.

22. Soini EJ, Hallinen TA, Puolakka K, Vihervaara V, Kauppi MJ. Cost-effectiveness of adalimumab, etanercept, and tocilizumab as first-line treatments for moderate-to-severe rheumatoid arthritis. J Med Econ. 2012;15(2):340-51.

23. Nishimoto N, Miyasaka N, Yamamoto K, Kawai S, Takeuchi T, Azuma J. Long-term safety and efficacy of tocilizumab, an anti-IL-6 receptor monoclonal antibody, in monotherapy, in patients with rheumatoid arthritis (the STREAM study): evidence of safety and efficacy in a 5-year extension study. Ann Rheum Dis. 2009;68(10):1580-4.

24. Bonafede M, Fox KM, Watson C, Princic N, Gandra SR. Treatment patterns in the first year after initiating tumor necrosis factor blockers in real-world settings. Adv Ther. 2012;29(8):664-74. 
25. Soliman MM, Hyrich KL, Lunt M, Watson KD, Symmons DP, Ashcroft DM, et al. Rituximab or a second anti-tumor necrosis factor therapy for rheumatoid arthritis patients who have failed their first anti-tumor necrosis factor therapy? Comparative analysis from the British Society for Rheumatology Biologics Register. Arthritis Care Res (Hoboken). 2012;64(8):1108-15.

26. Harnett J, Wiederkehr D, Gerber R, Gruben D, Koenig A, Bourret J. Real-world evaluation of TNFinhibitor utilization in rheumatoid arthritis. J Med Econ. 2016;19(2):91-102.

27. Bonafede MMK, Curtis JR, McMorrow D, Mahajan $\mathrm{P}$, Chen C-I. Treatment effectiveness and treatment patterns among rheumatoid arthritis patients after switching from a tumor necrosis factor inhibitor to another medication. Clinicoecon Outcomes Res. 2016;8:707-15.

28. Chastek B, Becker LK, Chen CI, Mahajan P, Curtis JR. Outcomes of tumor necrosis factor inhibitor cycling versus switching to a disease-modifying anti-rheumatic drug with a new mechanism of action among patients with rheumatoid arthritis. J Med Econ. 2017;20(5):464-73.

29. Wei W, Knapp K, Wang L, Chen CI, Craig GL, Ferguson $\mathrm{K}$, et al. Treatment persistence and clinical outcomes of tumor necrosis factor inhibitor cycling or switching to a new mechanism of action therapy: real-world observational study of rheumatoid arthritis patients in the united states with prior tumor necrosis factor inhibitor therapy. Adv Ther. 2017;34(8):1936-52.

30. Gottenberg JE, Brocq O, Perdriger A, Lassoued S, Berthelot JM, Wendling D, et al. Non-TNF-targeted biologic vs a second anti-TNF drug to treat rheumatoid arthritis in patients with insufficient response to a first anti-TNF drug: a randomized clinical trial. JAMA. 2016;316(11):1172-80.
31. Rotar Z, Hocevar A, Rebolj Kodre A, Praprotnik S, Tomsic M, Slovenian R. Retention of the secondline biologic disease-modifying antirheumatic drugs in patients with rheumatoid arthritis failing one tumor necrosis factor alpha inhibitor: data from the BioRx.si registry. Clin Rheumatol. 2015;34(10):1787-93.

32. Kim HL, Lee MY, Park SY, Park SK, Byun JH, Kwon S, et al. Comparative effectiveness of cycling of tumor necrosis factor-alpha (TNF-alpha) inhibitors versus switching to non-TNF biologics in rheumatoid arthritis patients with inadequate response to TNFalpha inhibitor using a Bayesian approach. Arch Pharm Res. 2014;37(5):662-70.

33. Harrold LR, Reed GW, Magner R, Shewade A, John A, Greenberg JD, et al. Comparative effectiveness and safety of rituximab versus subsequent anti-tumor necrosis factor therapy in patients with rheumatoid arthritis with prior exposure to antitumor necrosis factor therapies in the United States Corrona registry. Arthritis Res Ther. 2015;17:256.

34. Erkan D, Yazici Y, Harrison MJ, Paget SA. Physician treatment preferences in rheumatoid arthritis of differing disease severity and activity: the impact of cost on first-line therapy. Arthritis Rheum. 2002;47(3):285-90.

35. Rashid N, Lin AT, Aranda G Jr, Lin KJ, Guerrero VN, Nadkarni A, et al. Rates, factors, reasons, and economic impact associated with switching in rheumatoid arthritis patients newly initiated on biologic disease modifying anti-rheumatic drugs in an integrated healthcare system. J Med Econ. 2016;19(6):568-75.

36. Mathews AL, Coleska A, Burns PB, Chung KC. Evolution of patient decision-making regarding medical treatment of rheumatoid arthritis. Arthritis Care Res. 2016;68(3):318-24. 